\title{
THEMATISCHER SCHWERPUNKT 2012: Germanistik morgen
}

Die Germanistik in Polen und nicht nur in Polen, die Germanistik in ganz (Ost-)Mitteleuropa steht angesichts großer Umwälzungen vor völlig neuen Herausforderungen. Nicht nur, dass die Umstellung der Schullehrpläne zu einer Absenkung des sprachlichen Eingangsniveaus der Studienanfänger führt und das traditionell hohe Niveau des philologisch anspruchsvollen Germanistikstudiums zu unterlaufen droht, das - politisch geförderte - Übergewicht des Englischen in Wirtschaft, Politik und Medien unterstützt in Bewusstsein und Handeln verantwortlicher Funktionäre und Bildungsplaner in den Ministerien den Eindruck der Zweitrangigkeit der deutschen Sprache (für Literatur und Kultur schwindet unter dem Diktat ökonomischer Betrachtungsweisen ohnehin langsam das Verständnis). Das Interesse am Deutschen geht zurück. Dies gilt sogar in Regionen, in denen aufgrund unmittelbarer geographischer Nähe zum deutschen Sprachraum das Deutsche im Alltag, in Wirtschaft und Nachbarschaftskultur nach wie vor unmittelbar wichtiger und präsenter ist als das Englische, das insgesamt freilich unbestreitbar in die Rolle einer neuen Lingua franca auch in Mitteleuropa gerückt ist.

Zum Problem des sinkenden Eingangsniveaus von Studienanfängern im Fach Germanistik kommt auch deshalb an einigen Orten bereits jetzt das Problem sinkender Bewerberzahlen hinzu; verschärft wird dieses durch den demographischen Wandel, den langsam sich auf die Studienbewerberzahlen sich auswirkenden kontinuierlichen Geburtenrückgang und die gestiegene Mobilität, die es an Germanistik interessierten jungen Menschen erleichtert, gleich direkt in Deutschland oder Österreich zu studieren. Einige Universitätsgermanistiken haben sich in dieser Situation entschieden, auch Bewerber ohne Deutschkenntnisse aufzunehmen, was zu einer Revision der Studienpläne führt, im Mittelpunkt der Curricula steht vor allem der Sprachunterricht, das Angebot an literatur-, kultur- und sprachwissenschaftlichen Lehrveranstaltungen wird immer geringer.

Die Konkurrenz vorrangig sprachdidaktisch und fach- bzw. wirtschaftssprachlich orientierter Studiengänge an privaten Hochschulen (und Lehrercollegs) ist im Vergleich zu diesen grundlegenden Problemen fast schon marginal, solange die staatlichen Hochschulen ihren - wiederum von ihrem wissenschaftlichen Niveau abhängigen - Ruf zu wahren wissen, sie alleine könnten für eine wirklich vollwertige Ausbildung der Studierenden bürgen. Doch zeigt dies, dass das zumeist klar konturierte fachliche Profil der staatlichen Universitätsgermanistiken angesichts der Herausforderungen nicht 
einfach aufgegeben werden kann - sie würden mit ihm ihre Unterscheidbarkeit verlieren, das Fach den intellektuellen Anreiz, den es auf die besseren Studierenden nach wie vor auszuüben weiß - und die jeweiligen Länder einen beträchtlichen Teil ihrer auf Deutschland bezogenen Kompetenz. Zur Gefährdung dieser Unterscheidbarkeit tragen in Polen nicht zuletzt auch die sog. „ministerialen Standards“ bei, d.h. Mindestanforderungen, die vom Ministerium für das Hochschulwesen bestimmt wurden, denen jeder Studiengang entsprechen muss. Diese Mindestanforderungen lassen sehr wenig Spielraum bei der Gestaltung der Studienpläne, in die immer weniger von dem wissenschaftlichen Ertrag des jeweiligen germanistischen Instituts, einfließen ' kann. Auf die germanistischen Studiengänge wirkten sich selbstverständlich (es wird heftig diskutiert, ob positiv oder negativ) auch die Veränderungen aus, die durch den Bologna-Prozess erzwungen wurden.

Unter diesen Voraussetzungen müssen Antworten auf die an unterschiedlichen Orten bereits unterschiedlich deutlich gewordene existentielle Krise gefunden werden, die keine modisch mit intellektuellem Gewinn vortragbare Krise mehr ist wie jener lange gepflegte Methodenstreit, der es insbesondere der Muttersprachengermanistik in Deutschland selbst lange erlaubt hatte, sich in den Feuilletons öffentlich um sich selbst zu drehen. Diese Krise geht an die Substanz des Faches in vielen Ländern und wirft grundsätzliche Fragen auf: Wie soll auf das gesunkene Anfängerniveau reagiert werden? Wie gewinnt man überhaupt Studierende? Wie lassen sich zumeist literatur- und philologiefern sozialisierte junge Menschen noch für ein philologisches Studium begeistern? Ist die für die fachliche Identität und Erkennbarkeit des Faches so wichtige philologische Tradition eher Hilfe oder eher Ballast? Diese Probleme betreffen jede Germanistik in Polen und in ganz (Ost-)Mitteleuropa und treffen sie doch aufgrund ihrer jeweiligen Verfasstheit anders, jede kann und muss aufgrund ihrer jeweiligen Besonderheiten, ihrer Strukturen, ihrer personellen Zusammensetzung, ihrer regionalen Einbindung und ihrer fachlichen Traditionen auf jeweils ihre besondere Weise reagieren. Hin und wieder freilich lassen sich überregional wirksame Muster bereits jetzt erahnen. An vielen Orten wird mit Veränderungen der Lehrpläne, mit der Einführung von Anfängerkursen auf Nullniveau, aber auch grundlegenden Änderungen der Studienfachprofile experimentiert. PR-Maßnahmen kommen zu den ohnehin nicht geringen Aufgaben des wissenschaftlichen Personals hinzu, mancherorts kann man bereits bisher nur von privaten Hochschulen bekannte Plakate und Banner sehen, die zum Studium neu profilierter germanistischer Studiengänge nun auch an staatlichen Universitäten einladen. 
Thematischer Schwerpunkt 2012

Über den von den jeweils konkreten Umständen vor Ort geprägten Einzelversuchen droht freilich gelegentlich der Überblick über die schwierige Gesamtlage verlorenzugehen. CONVIVIUM möchte deshalb dazu einladen, sich darüber auszutauschen - grundsätzliche Überlegungen zur Lage des Faches in Mitteleuropa sind ebenso notwendig, wie Informationen über konkret einzelne Reaktionen am jeweiligen Hochschulstandort insbesondere in Polen willkommen. Wie wird mit der neuen Situation umgegangen, welche Lösungen werden ihr abgerungen?

Themenvorschläge werden erbeten bis 30. Juni 2011, ausgearbeitete Beiträge bis zum 30.11.2011. Die „Hinweise zur Einrichtung des druckfertigen Manuskripts" können unter www.convivium.pl eingesehen werden. 